Nouvelles perspectives en sciences sociales

\title{
Sur la méthode topico-critique : au service de la reconstruction scientifique
}

\section{Jean-Louis Le Moigne}

Volume 2, numéro 2, mars 2007

URI : https://id.erudit.org/iderudit/602458ar

DOI : https://doi.org/10.7202/602458ar

Aller au sommaire du numéro

\section{Éditeur(s)}

Prise de parole

\section{ISSN}

1712-8307 (imprimé)

1918-7475 (numérique)

Découvrir la revue

\section{Citer cet article}

Le Moigne, J.-L. (2007). Sur la méthode topico-critique : au service de la reconstruction scientifique. Nouvelles perspectives en sciences sociales, 2(2), 13-31. https://doi.org/10.7202/602458ar
Résumé de l'article

S’interroger sur la légitimité des propositions scientifiques que nous élaborons et enseignons apparaît souvent comme une " incongruité » dans le contexte épistémique de l'époque. Pourtant une riche tradition de la pensée occidentale qui va de Protagoras à Morin en passant, entre autres, par Vico, Bachelard et Piaget, nous alerte contre l'illusion d'un savoir divin universellement certain. La " fin des certitudes ", l'enracinement de la connaissance et l'interrogation du souhaitable à partir du faisable constituent des principes alternatifs à l'épistémologie dominante capables de fonder le travail de légitimation des connaissances que nous produisons. 


\title{
Sur la méthode topico-critique : au service de la reconstruction scientifique ${ }^{1}$
}

Topica cum critica conjunctura.

\author{
JeAN-LOUIS LE MoIGNE \\ Université Paul Césanne d'Aix-Marseille
}

Commençons par complexifier! Peut-on ouvrir cette réflexion sur les " méthodes d'études (et d'enseignement) de notre temps ${ }^{2}$ » par cette formule provocante? Chacun se souvient de la recette préférée de tous les systèmes d'enseignement et de toutes les vulgarisations, celles qu'on utilise pour «le vulgaire » : « commençons par simplifier», autrement dit «à diviser (la matière, qu'elle soit première à extraire, ou seconde, à enseigner) en autant de parcelles qu'il se pourrait ", puis à aligner ces parcelles " en longues chaines de raisons toutes simples et faciles $»$. Recette infaillible, diton, puisque le Discours de la méthode, pour chercher la vérité dans les sciences, nous assure " que toutes les choses qui peuvent tomber sous la

1 J'emprunte ce titre et l'exergue aux intitulés des $\$$ II-2-2 et VI-4, p. 107, de l'ouvrage de Davide Luglio, I a science nouvelle ou l'extase de l'ordre. Connaissance, rbétorique et science dans l'xurre de G. B. Vico, Paris, PUF, 2003. Je m'en explique plus loin.

2 Reformulation du titre de l'ouvrage de Giambattista Vico, La méthode des études de notre temps, dans Vie de G. B. Vico écrite par lui-même, traduit de l'italien par Alain Pons, Paris, Grasset, [1708] 1981. 
connaissance des hommes s'entre-suivent en même façon ${ }^{3}$ ». Prendre si directement le contre-pied d'une recette que les académies ont souvent instituée en dogme, dans tous les domaines de la construction scientifique (qu'elles concernent les disciplines dures autant que les douces) et, en particulier, dans le plus surveillé, celui de l'éducation, n'est-ce pas là un geste incongru?

Mais comme « l'incongruité épistémologique ${ }^{4}$ » s'avère source féconde de solides réflexions critiques sur la légitimité socioculturelle des propositions scientifiques que nous élaborons et enseignons, nous pouvons nous risquer aujourd'hui sans grand danger à cette large ouverture de l'éventail de nos méthodes de recherches et d'enseignements. La riche histoire des sciences nous donne assez d'exemples de la fécondité de cette ouverture épistémo-méthodologique pour que l'on ne puisse nous accuser de sombrer dans un délire onirique ou dans un charlatanisme.

Nous introduisant à la méthode de Léonard de Vinci, Paul Valéry nous invitait à nouveau à en convenir : "Celui qui se représente un arbre est forcé de se représenter un ciel ou un fond pour l'y voir s'y tenir ${ }^{5} »$. Pour représenter l'arbre, au lieu de d'abord le séparer de son contexte, commençons par les conjoindre : car nous ne savons les percevoir et les comprendre que dans leurs innombrables interactions. Autrement dit, commençons par complexifier notre image de l'arbre pour tenter d'en former quelque entendement : complexifier, c'est d'abord conjoindre, intentionnellement. Alors que simplifier, c'est d'abord disjoindre peut-être sans nécessité.

"Complexifier c'est conjoindre », belle devise qui exprime le profond respect du modélisateur pour l'intelligence de ses lecteurs. Je conserve le

3 Faut-il rappeler ici les références précises des quatre préceptes du Discours de la méthode de Descartes publiés en français en 1637 ?

4 J'emprunte ici l'expression à Yves Barel, Le paradoxe et le système. Dix ans après, Grenoble, Presses univesitaires de Grenoble, 1989, p. 315. Quelques précisions dans Jean-Louis Le Moigne, Le constructivisme. Modéliser pour comprendre, tome 3, Paris, L'Harmattan, 2004, p. 238.

« Introduction à la méthode de Léonard de Vinci », repris dans l'édition « La pléiade " (Euures completes, tome 1, Paris, Gallimard, [1894] 1974, p. 1156). La métaphore de la modélisation de l'arbre se poursuivra longtemps dans les méditations de Paul Valéry : en 1943, il publiait un merveilleux Dialogue de l'arbre, repris dans « La pléiade », tome 2,1957 ,p. 177, qui nous dit l'arbre dans ses contextes temporels plus encore que dans ses contextes spatiaux. 
souvenir amer des propos d'un dirigeant d'industrie, représentant éminent de «l'élite républicaine», intitulant son appel, tenu en 1986, Le retour du bon sens. Mobiliser les hommes, c'est simplifier les systemes. Titre qu'il justifiait par l'argument «Les gens (les autres, pas lui) ne sont jamais que des êtres humains », et pour eux, il vaut mieux « simplifier ». Ceci sans percevoir un seul instant que rien n'était plus complexe que ce qu'il tenait pour simplification : «Déléguer l'autonomie, devenir grand tout en restant petit, se distinguer des autres par l'attention portée au client ${ }^{6} »$. Rien de plus simple? Le croyez-vous vraiment?

C'est en lisant de tels propos que j'ai pris conscience de la dramatique duperie éducative que symbolisent aujourd'hui encore «les quatre préceptes du discours cartésien » ou «l'unique devise qui convienne à la politique [...] Ordre et progrès », proposée par Auguste Comte, « devise qui caractérise la conciliation... réalisée dans le positivisme ${ }^{7} »$.

L'appel à la simplification, au « simplifier d'abord» ou à l'usage universel d'un « rasoir d'Ockam ${ }^{8} »$, n'est pas nécessairement la meilleure recette sur

- Article de Claude Jouven publié dans Le Monde du 8 avril 1986, sous le titre « XXI" siècle : le retour du bon sens, mobiliser les hommes, c'est simplifier les systèmes ".

Auguste Comte, "Appel aux conservateurs », publié dans Du pouvoir spirituel (choix de textes de Pierre Arnaud), Paris, Le livre de poche, [1855] 1978, p. 493-494.

8 Peut-être faut-il s'interroger sur les raisons historiques de cette priorité accordée trop systématiquement à ce principe universel de simplification. Elles sont en effet souvent argumentées par la référence à un principe de philosophie médiévale généralement présenté depuis un siècle comme quasi universellement scientifique, le « principe de parcimonie ", dit de Guillaume d'Ockham (1330), souvent appelé depuis 1918, «Principe du rasoir d’Ockham » bien que celui-ci n'ait jamais utilisé cette métaphore du rasoir ! : « Il ne faut pas multiplier les entités au-delà du nécessaire ». Ce principe, parfois présenté sous des noms variés tels que le principe d'économie, le principe de moindre action, le principe de parcimonie, le principe de simplicité, le principe (ou la loi) du moindre effort, le principe d'optimum, se présente comme une règle méthodologique dont la légitimité épistémique pourrait être tenue pour universelle. Il impliquerait alors une finalité unique et invariante par rapport à laquelle tout dans l'univers s'ordonnerait impérativement. De tous les modèles concevables d'un phénomène, celui respectant au mieux le principe de moindre action ou le principe de parcimonie universelle serait à la fois le plus certain épistémiquement et le plus simple (le plus économique en nombre de symboles computables) méthodologiquement. Cette réduction systématique du certain ou du vrai au simple (qui a fait la fortune de la méthode cartésienne dans toutes les sciences pendant deux siècles) n'a pas a priori de légitimité fondamentale. Parfois plausible, sans plus, elle ne s'impose pas à l'esprit humain raisonnant avec l'évidence d'un axiome universel, et 
laquelle puisse se construire un projet scientifique civique et moins encore un projet éducatif. "La simplification est dénaturation du réel par mutilation, réduction, et disjonction ", nous rappelait déjà Edgar Morin, la contrastant avec « la simplicité (celle de L'Idiot dostö̈evskien [...] celle du Sermon sur la montagne) qui est toujours l'émergence d'une fabuleuse complexité $»$.

Ne pouvons-nous prendre enfin au sérieux cet appel à l'intelligence de la "fabuleuse complexité » de notre relation au monde, au lieu de la trivialiser par simplification, sous le prétexte que nous (" les gens ») ne serions " que des êtres humains », présumés incapables d'intelligence, d'étonnement et d'émerveillement? Alors que nous avons la chance, inouie peut-être, de nous connaître êtres humains, existant, cogitant, agissant et devenant, souvent intentionnellement !

l'observation des phénomènes naturels suggère d'innombrables contre-exemples. La formulation initiale du principe de Guillaume d'Ockham peut, au demeurant, être interprétée en en renversant les termes : «Il ne faut pas multiplier les entités plus que de besoin » peut se lire : «Il faut multiplier les entités autant que de besoin », quitte à ce qu'ainsi on augmente le nombre de symboles et de patterns les associant. Le principe d'Ockham n'est plus alors celui d'un rasoir qui découpe, mais celui d'un projecteur qui, en éclairant, relie (ce qui incidemment semble bien plus fidèle à la pensée de Guillaume d'Ockam. Ce que nous pouvons interpréter en reconnaissant au principe de simplification a priori une vertu d'heuristique plausible à fin d'économic cognitive du modélisateur, mais nullement un caractère de nécessité fondamentale s'imposant a priori à la conception de tout modèle scientifique, que ce modèle porte sur la représentation des data ou sur les procédures de leur traitement. La distinction du moyen méthodologique (le principe de parcimonie ou de simplification) et de la fin éthique (le projet explicite du modélisateur) constitue la base de toute critique épistémologique interne. Le principe de parcimonie ne saurait constituer le critère de scientificité d'une "connaissance enseignable et actionnable ", à la différence du principe d'intelligibilité : il importe qu'un énoncé scientifique puisse être compris, il n'importe pas qu'il soit d'abord le plus simple possible.

9 Edgar Morin, dans Jean-Pierre Dupuy, Ordre et désordres. Enquête sur un nouveau paradigme, Paris, Seuil, 1982, p. 247-249. 


\section{Connaissances mimétiques, connaissances poïétiques}

I a connaissance n'est pas la pensée. La connaissance est un véritable « milieu »"

Relever ce défi, c'est bien sûr s'attacher à formuler et à légitimer des stratégies cognitives et des pratiques éducatives alternatives qui ne fassent plus de la complexité « le mal absolu » ou " une croyance moralement dangereuse $^{11}$ ", ni de la simplification l'unique et universel mode d'entendement de notre relation aux autres et à l'univers. Il ne s'agit pas, comme on l'a cru et enseigné trop souvent depuis 30 ans, d'ajouter une couche "système » à la couche " analyse " (pour faire "l'analyse de système »), ou d'ajouter une « causalité téléonomique » (aussi déterminante que la causalité efficace), à l'hypothèse déterministe érigée en principe exclusif, et ignorante des " projets du sujet modélisant l'objet ${ }^{12}$ ».

Ce sujet (vous et moi) n'est-il pas capable de symbolisation et de computation téléologique (finalisante plutôt que finalisée) ? N'est-ce pas à cette faculté, interrogeait Karl Marx, que nous reconnaissons la supériorité de l'architecte le plus banal sur l'abeille la plus experte ? : «Il construit la cellule dans sa tête avant de la construire dans la ruche ${ }^{13}{ }^{\prime}$.

Il faut assumer en effet l'abandon de l'exclusif impératif platonicien d'un savoir préétabli, détenu par « des hommes aussi bien que des femmes qui étaient savants dans les choses divines [...] qui tenaient un langage vrai $[\ldots]$ qui sont tous les prêtres ou prêtresses [...] tous ceux qui sont divins ». Un «savoir mimétique» tel que, «en fin de compte, chercher et apprendre sont dans leur entier une remémoration ${ }^{14} »$.

10) Paul Valéry, Cahiers 1894-1914, dans CEuvres complètes, Paris, Gallimard, coll. « La pléiade ", tome 2, p. 69.

1 Comme l'enseigne un professeur du département de comptabilité de l'école HECFrance : Voir Business Digest, juin 2002, accessible à

http: / $/$ bdt.businessdigest.fr $/$ frame $/$ lg $=$ fr\&sid $=\& p=14 \& q=\& \mathrm{th}=$ Economie $\% 20$ et $\% 20$ soci $\%$ E9t $\%$ E9\&sth=Soci $\% E 9 t \% E 9 \&$ do $=$ list.

12 Gaston Bachelard, «I a méditation de l'objet par le sujet prend toujours la forme du projet », Le nouvel esprit scientifique, Paris, PUF, 1934, p. 15.

1.3 Karl Marx, Le capital, dans (Euvres, tome 1, Économie, Paris, Gallimard, coll. " La pléiade », [1867] 1963, p. 728.

14 Platon, "Ménon ou de la vertu », dans CEurres complètes, Paris, Gallimard, coll. « Ia pléiade », p. 528-529. 
L'exercice n'est pas plus difficile que celui auquel nous nous étions accoutumés, rassurés peut-être fallacieusement par d'illustres cautions telles que Platon, Descartes, Newton ou Comte, tenus pour « savant dans les choses divines $)$.

Protagoras et Aristote (écrivant Les topiques et Les rbétoriques), Vico écrivant en 1708 son Discours de la méthode des études de notre temps ${ }^{15}$ (pour balancer l'audience du discours cartésien qui commençait à se diffuser en Occident), et, plus proches, Valéry, Bachelard (Le nouvel esprit scientifique et son appel à une « épistémologie non cartésienne » paraît en 1934), Piaget, Simon $^{16}$, Morin, tant d'autres non moins illustres, bien qu'ils ne se targuent pas d'être savants dans les choses divines, témoignent par leurs œuvres de la faisabilité des « connaissances poḯtiques ».

L'exercice n'est pas plus difficile, mais, pour le pratiquer, il fallait d'abord résister à la pression des méthodologues de toutes obédiences. Indifférents aux référents épistémiques des recettes qu'ils utilisent ou enseignent, ceux-ci ne consentent à changer ces recettes (qui ne sont que d'application

15 La traduction-présentation de cet « autre discours de la méthode », due à Alain Pons, est désormais disponible sur Internet à

http://www.mcxapc.org/docs/conseilscient/0511 vico_pons.pdf.

16 À bien des reprises, Herbert A. Simon a insisté sur cette faisabilité computationnelle et cognitive des connaissances poïétiques, en soulignant ce qu'il appelait heureusement « le paradoxe du Ménon ». La discussion du Paradoxe du Ménon et de l'interprétation qu'en donne Platon constitue l'un des arguments pivot de sa réflexion épistémologique. Outre les commentaires qu'il développe dans sa "Conférence Turing » de 1975, il y reviendra peu après dans une note publiée en 1976 dans Philosophy of science ( $\mathrm{n}^{\prime \prime}$ 43, p. 147-151), et reprise dans Models of Discovery (1977, chapitre 5.5 : «Discussion : The Meno paradox »). Cet article est traduit en français par Dan Sperber et publié dans Monique Canto-Sperber (dir.) (Les paradoxes de la connaissance. Essai sur le Menon de Platon, Paris Odile Jacob, 1991, p. 329-333). Le traducteur et l'éditeur français ne mentionnent malheureusement pas le contexte dans lequel cette brève note de Simon doit être interprétée, en ignorant en particulier la discussion épistémologique argumentée qu'il consacre à la thèse platonicienne du Menon et à la théorie de la computation dans sa "Conférence Turing », publiée la même année que la note intitulée "Discussion: The Meno paradox ». J'ai développé ces arguments dans l'article «Sur un exceptionnel manifeste épistémologique : Symbols and Search; Merveilleuse et pourtant compréhensible est la computation (le traitement) heuristique des systèmes de symboles physiques ", publié dans La revue d'intelligence artificielle, numéro spécial en hommage à H. A. Simon, 2002 (Représentations, découvertes et rationalité, sous la direction de Jacques Pitrat, Hermés-Lavoisier, RSTI, série RIA,

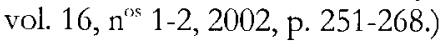


mimétique de savoirs de type platonicien) qu'à la condition que ne changent pas ces référents qui devraient les légitimer.

Aussi sachons gré aux chercheurs contemporains qui s'attachent à la critique épistémique de leurs travaux, de savoir résister à la pression académique qui les incite, aujourd'hui encore, à faire commerce des théories de la complexité, du chaos et des systèmes non linéaires, sans autres justifications que celle d'un mimétisme platonicien! En s'attachant à entendre l'avertissement que Giambattista Vico adressait, il y a trois siècles, aux premiers dévots du Discours de la méthode: "Car la méthode nuit à l'ingenium, et l'ingenium a été donné aux humains pour comprendre, c'est-àdire pour faire ${ }^{17}$ », ils nous aident à revitaliser l'aventure de la connaissance en l'insérant au cœur de l'aventure humaine.

C'est, je crois, le grand mérite de ces chercheurs contemporains que d'assumer tranquillement la complexité de leur projet. En s'attachant à expliciter les référents épistémiques sur lesquels peuvent se légitimer, ici et maintenant, les initiatives, les actions et surtout les évaluations des projets scientifiques et éducatifs qu'appelle le défi contemporain de la complexité, ils nous rendent un réel service culturel. Ils ne retiennent peut-être pas longtemps l'attention des lecteurs qui ne veulent connaitre que des recettes qui marchent sans que l'on ait en plus à réfléchir à leur mise en œuvre dans des contextes changeant? En revanche, ils gagneront celle des lecteurs qui s'attachent « à comprendre pour faire autant qu'à faire pour comprendre ». Autrement dit, à tous ceux qui s'attachent à légitimer leurs enseignements, leurs recherches et leurs actes éducatifs, sans se résigner à l'application de recettes élaborées avant et ailleurs, dans des contextes et à des fins qu'ils ignorent.

En renonçant aux multiples simplifications qu'appelle l'apprentissage mimétique de savoirs légitimés par « celles et ceux qui sont savants dans les choses divines » (en langage contemporain, on dira souvent : "par les académies »), on s'oblige à s'interroger, avec " une obstinée rigueur » (Hostinato rigore, la devise que Léonard de Vinci plaçait en exergue de ses Carnets), sur ce que peut être une connaissance poïétique : "Toute connaissance acquise sur la connaissance devient un moyen de connaissance éclairant la connaissance qui a permis de l'acquérir ». La définition récursive

17 Giambattista Vico, De l'antique sagesse de l'ltalie, traduction de Jules Michelet, Paris, Garnier-Flammarion, [1710]1993, p. 128-136. Je n'ai pas repris la formule de Michelet : « la méthode entrave le génie », révélatrice de la difficulté de trouver un équivalent français au mot ingenium. 
qu'en propose Edgar Morin en conclusion de La connaissance de la connaissance $^{18}$ synthétise les termes de ce stimulant défi, celui de «l'intelligence de la complexité19 $\%$.

Stimulant en ceci que chacun a l'expérience cognitive sensible et familière de cette connaissance poïétique, qu’il apprenne avec Ménon ce qu'est la Vertu, ou avec l'instituteur, ce qu'est une multiplication. Et, en même temps, chacun s'inquiète : "Comment vais-je légitimer cette connaissance transformante que je me construis et que j'enseigne ou que j'apprends? À quel moment saurai-je que cette connaissance devient Bateau ivre, délire onirique?»

S'il n'est plus nul « grand prêtre (ou nul expert scientifique) savant dans les choses divines » qui puisse cautionner universellement nos connaissances (quel que soit leur mode d'acquisition, transmise par un maitre ou auto-écoproduite au fil de nos propres activités cognitives), il nous reste, pour les légitimer, l'ascèse intellectuelle que peut exercer chaque citoyen et le pragmatique usage de la délibération démocratique.

Pyrrhon, Protagoras, Aristote par Les topiques et Les rhétoriques, Cicéron, Montaigne, Pascal, Vico... Ne sont-ils pas légions dans nos cultures, ceux qui nous ont accoutumé à ces exercices du «bon usage de la raison dans les affaires humaines » en assumant sagement « la fin des certitudes ${ }^{20}{ }^{2}$ »?

\section{La méthode topico-critique au service de la reconstruction scientifiques $^{21}$}

Car s'il n'est nul savoir divin universellement certain sur lequel nous puissions fonder et légitimer les connaissances que nous produisons, enseignons, apprenons et actionnons, nous avons la chance considérable de pouvoir atténuer sensiblement les risques de leur dégénérescence fatale en un délire onirique ou cauchemardesque. Nous pouvons prêter attention à quelques-uns des critères de légitimation épistémologiques des connais-

18 Edgar Morin, La métbode, tome 3, L a connaissance de la connaissance, Paris, Seuil, 1986, p. 232.

19) Edgar Morin et Jean-I _ouis Le Moigne, L'intelligence de la complexité, Paris, L'Harmattan, 1999.

20) J'emprunte ici le titre de l'ouvrage d'Ilya Prigogine, I a fin des certitudes, Paris, Odile Jacob, 1996.

21 Davide Luglio, I a science nouvelle..., op. cit., p. 107. 
sances enseignables que nous livrent ces sages. Connaissances plutôt que savoirs, connaissances réflexives qui sont toujours « réorganisation critique de la connaissance ».

Critères de circonstance (comme le sont ceux du « discours cartésien ${ }^{22}$ », 1637, ou du « catéchisme positiviste » comtien, 1852), aujourd'hui pragmatiquement entendu dans toutes les cultures humaines dès lors que nous nous astreignons à les expliciter loyalement. Il y a en effet un démagogique abus de langage dans la récente proclamation d'un « pacte républicain refondé au début de la $\mathrm{III}^{\mathrm{e}}$ République par Jules Ferry... déléguant à une institution, l'Université, la mission de faire respecter le principe du partage entre une vérité scientifique et des opinions ${ }^{23}{ }^{\prime}$. Que cette convention platonicienne ait été tacitement acceptée en Occident en une période de scientisme positiviste triomphant ne lui vaut aucune valeur éternelle! (Elle n'est d'ailleurs inscrite dans aucune constitution.) Comme toute convention qui ne se régénère pas, elle dégénère vite en une « épistémologie d'État souverainiste » qui interdit toute discussion avec les citoyens, lesquels sont présumés obscurantistes bien qu'ils financent la recherche scientifique.

Sans doute faut-il quelque sorte de bannière qui symbolise assez la convention épistémo-méthodologique à laquelle chacun se réfère pour argumenter intelligiblement la légitimité des connaissances qu'il construit et enseigne. Ne peut-on désacraliser (sans la sataniser) "la convention analytico-syllogistique » qui refondait au siècle dernier le «pacte républicain science-société » et que garantirent longtemps les institutions académiques (en Europe surtout), dès lors que les sociétés peuvent former une autre convention épistémologique plus ouverte, et tenue pour mieux argumentée dans nos cultures?

On peut, je crois, retenir aujourd'hui l'une des formulations de Vico pour la désigner de façon significative, à la fois adaptée aux conditions pragmatiques et éthiques de la recherche scientifique contemporaine, et au moins aussi bien enracinée dans nos cultures. Aristote ici nous sert de solide repère puisque nous lui devons tous à la fois Les anabytiques (et leur

22 "Un discours sur la méthode scientifique sera toujours un discours de circonstance, il ne décrira pas une constitution définitive de l'esprit scientifique ", Gaston Bachelard dans Le nomel esprit scientifique (Paris, PUF, 1934, p. 139).

23 Extrait d'un article de François Ewald et Dominique Lecourt, « Les OGM et les nouveaux vandales », Le Monde, 4 septembre 2001. 
instrument, la syllogistique parfaite) et I es topiques, avec la rhétorique (et leur instrument, la méthode dialectique ${ }^{24}$. En nous proposant de dépasser l'analytique syllogistique (ou cartésienne) exclusive et en nous invitant à déployer l'éventail sans en exclure un brin par la restauration de «la Topique éclairée par le flambeau de la Critique ", Vico nous invite à assumer pragmatiquement la complexité de toute méthode d'enquête scientifique : «Mais si l'on parcourt avec le flambeau de la critique tous les lieux de la topique, alors on sera certain de connaitre l'objet... proposé. [...] Et dans cet examen successif, la topique même est critique ${ }^{25} \%$.

Luglio montre ce que " cette conception de la topique comme instrument épistémologique » développée par Vico doit aux arguments de "François Bacon, Baron de Verulam, homme d'une sagesse incomparable »: « Car ce que l'on cherche, c'est par une seule et même opération de l'esprit qu'on l'invente et qu'on le juge $e^{26} »$.

Cette discussion très approfondie de la pensée de Vico conduit Luglio à proposer une formule qui me semble fort bienvenue pour servir d'emblème à notre convention épistémo-méthodologique : « la méthode topico-critique au service de la reconstruction scientifique ${ }^{27} \gg$.

$\mathrm{Si}$, pour donner un tour plus contemporain à cet intitulé, on souhaite retrouver des formulations plus contemporaines, je proposerai volontiers la conjonction de " la modélisation systémique » et de "la rationalité procédurale », comme aussi bien le titre aujourd'hui plus familier sous lequel Edgar Morin a campé l'essentiel de La méthode: « la pensée complexe ». Intitulés qui nuancent les divers éclairages à la fois poïétique et critique sous lesquels on examine «toutes les questions que l'on peut élever sur l'objet proposé $»$.

24 Le classique de Jean-Marie Le Blond, Logique et méthode chez. Aristote (Paris, Vrin, [1939] 1970) met remarquablement en valeur la riche complexité de l'apport d'Aristote à la formation des conventions épistémologiques dans nos cultures.

25. Giambattista Vico, De l'antique sagesse de l'Italie [1710], traduction de Jules Michelet, présentation de Bruno Pinchard, Paris, Garnier-Flammarion, 1993, p. 125.

26 Davide Luglio, I a science nouvelle..., op. cit., 2003, p. 37-38.

2? Ibid., p. $107-111$. 


\section{Les critères contemporains de légitimation des connaissances enseignables}

Travaillons donc à bien penser: voilà le principe de la morale ${ }^{28}$.

Sous ces labels se forment et se transforment des critères ou des repères guidant téléologiquement les évaluations des "jugements » et les explorations des domaines ainsi délibérément éclairés. Repères qui inscrivent l'aventure de l'humaine connaissance dans l'inséparable conjonction de l'expérience, la pragmatique, de la conscience, l'éthique, et de la science, l'épistémique. D'abord conjoindre : la pragmatique appelle l'éthique qui appelle l'épistémique qui appelle la pragmatique qui appelle...

La devise de Pascal ne nous dit-elle pas cette antique sagesse pouvant guider toutes nos méthodes de construction et de reconstruction des connaissances enseignables ? : «Travaillons donc à bien penser : voilà le principe de la morale ${ }^{29}$ ».

\subsection{Le critère de fin des certitudes}

Le premier critère fonctionne négativement : toute connaissance qui ne serait intelligible qu'en se présentant comme universellement certaine ne peut être tenue en tant que telle pour légitimement enseignable. Ce serait le cas, par exemple, de connaissances astrologiques ou de la théorie de la purification ethnique. Elles peuvent tenter de se présenter comme incertaines bien que localement plausibles, mais elles cessent d'être connaissances enscignables dès qu'elles s'imposent comme universellement et éternellement certaines. L'intérêt considérable du critère d'incertitude est qu'il contraint désormais les sociétés humaines à afficher explicitement leurs options éthiques, au lieu de déléguer subrepticement ces choix collectifs aux scientifiques producteurs de connaissances scientifiques, lesquels oublient parfois qu'elles ne sont pas absolument certaines.

28 Blaise Pascal, Pensées, 200-347 H3, Paris, Seuil, 1963.

29) «L'homme n'est qu'un roseau, le plus faible de la nature, mais c'est un roseau pensant. [...] Toute notre dignité consiste donc en la pensée. C'est de là qu'il nous faut relever et non de l'espace ou de la durée que nous ne saurions remplir. Travaillons donc à bien penser : voilà le principe de la morale ». dans Pascal, op.cit., p. 528 de l'édition Lafuma. 


\subsection{Le critère d'enracinement}

Le second critère fonctionne plus positivement : si elle n'a pas de " fondement au sens maçon et architectural du terme », la connaissance a des « enracinements ». « La connaissance humaine [...] peut s'originer [...] en un poly-enracinement anthropologique (cérébral, spirituel, culturel, social), lequel nécessite un poly-enracinement physique / biologique / zoologique [...] (autrement dit en) un principe de l'auto-éco-organisation vivante qui comporte en elle-même la dimension cognitive $\left.e^{30}\right)$.

Ce critère de l'enracinement de la connaissance dans l'histoire - ou dans l'aventure - humaine (qui nous rend intelligibles les connaissances produites par une humanité qui est "son œuvre à elle-même » et qui peut donc " connaitre " ses propres cuvres, selon le mot de Vico) s'avère pragmatiquement très fécond. Il nous protège des connaissances " qui tombent du ciel » produites par quelques illuminés, et surtout, il contraint les scientifiques et les enseignants à ne plus séparer « les deux cultures » (la culture dite scientifique et la culture tout court que l'on appelait les humanités).

" Mainte erreur, gâtant les jugements qui se portent sur les cuvres humaines, est due à un oubli singulier de leur génération ${ }^{31}$ ", nous rappelle Valéry. Toute connaissance, pour être légitime, doit reconnaitre ses propres " enracinements ». "Ces conjonctions fabuleuses de conditions physiques, électriques, chimiques, neuronales, cérébrales, computantes, cogitantes, spirituelles, linguistiques, culturelles, sociales pour que se constitue et se perpétue un "simple" savoir ${ }^{32} »$. Toute connaissance s'enracine dans les connaissances qui ont permis de l'acquérir, qu'elle éclaire en retour.

L'important est dans la conjonction de cette " reconnaissance de paternité» et de sa critique épistémologique explicite. L'exemple des lectures du Ménon est significatif. Nombre de chercheurs et d'enseignants se réfèrent implicitement à une épistémologie platonicienne pour légitimer leurs arguments. Oseront-ils enfin nommer « les hommes et les femmes qui étaient savants dans les choses divines... » et qui les ont assurés que les énoncés qu'ils produisaient étaient "vrais " puisque eux même s'en

30) Edgar Morin, La méthode, tome 3, op. cit., p. 231.

31 Paul Valéry, «Introduction à la méthode de Léonard de Vinci », op. cit., p. 1157.

32 Edgar Morin, La métbode, tome 3, op .cit., p. 231. 
souvenaient ? Conviendront-ils qu'ils se sont résignés à un bien peu scientifique principe d'autorité, en se soumettant à «tel créateur scientifique de génie, comme Descartes ou Leibniz, [qui] laisse là pour un temps ses travaux spécialisés, et s'adonne à la construction d'une philosophi ${ }^{33}$ », ou comme Auguste Comte qui s'était sans rire auto-institué «fondateur et grand prêtre de la religion universelle, religion de l'humanité », garantie symbolique de leur « savoir dans les choses divines »?

Ce rappel n'est pas fréquent et les académies ne le préconisent pas volontiers, craignant à juste titre que ne soit révélée au public la légèreté de certains des fondements des vérités scientifiques qu'elles devraient certifier. Car enfin la vérité universelle de « la loi des trois états » de Comte ${ }^{34}$ ou du solipsisme cartésien " Je pense donc je suis " n'est pas toujours aussi convaincante qu'elle le devrait. Si les fondements sont reconnus fragiles, que sera la solidité de l'édifice? Ne faut-il pas prévenir les usagers (et les enseignés) que ces connaissances certifiées ne sont pas toutes également assurées, et rappeler l'origine de cette assurance ? N'est-ce pas là que devrait commencer l'exercice de cet esprit critique dont les scientifiques veulent heureusement faire vertu?

En veillant à rappeler la genèse critique des connaissances qu'ils produisent ou transmettent, en témoignant de leur permanente attention à "l'histoire et à la philosophie des sciences", ils organisent une sorte de " base de départ » de cette " critique épistémologique interne [...] [qui] devient alors $[\ldots]$ organisation intérieure des fondements ${ }^{35} "$, selon le mot de Piaget en 1967. Aujourd'hui, nous parlerons plus volontiers de « l'autoéco-organisation productrice des connaissances humaines ».

33 Jean Piaget, "Logique et connaissance scientifique ", Encyclopédie Pléiade, Paris, Gallimard, 1967, p. 51.

34 En 1990 encore, René Thom, à court d'arguments convaincants pour l'emporter dans « la querelle du déterminisme » qu'il avait suscitée, pouvait écrire : « Pour le montrer, il est bon de revenir à la vieille loi comtiste des trois états » (L a querelle du déterminisme, Paris, Gallimard, coll. «Le débat», p. 267), sans s’interroger sur la légitimité de cette présumée loi!

35 Jean Piaget, op. cit., p. 51. 
3.3. Le critère d'intelligibilité par le «faire-comprendre »

Une fureur sacrée de « comprendre pour faire » et de "faire pour comprendre », qui passe toute philosophie $e^{36}$.

Le troisième critère sera celui du « faire-comprendre ». Par ce néologisme, on peut synthétiser l'argument vicéen du Verum et factum reciprocantur ( $\mathrm{La}$ vérité est dans le faire » ou, traduction de Jules Michelet, «le vrai et le fait se mettent l'un pour l'autre »), qui ne confond plus le vrai et le bien, mais qui permet d'interroger sans cesse le souhaitable (le bien) à partir du faisable (le vrai).

La connaissance enseignable s'entend alors parce qu'elle permet de comprendre et de faire comprendre : la connaissance des faits devient la connaissance des faire (ou la reconnaissance du fait qu'un « fait» [tenu pour un « objet»], pour être fait, doit pouvoir se « faire » intentionnellement, par "projet»). «Connaittre ne consiste pas à copier le réel, mais à agir sur lui et à le transformer (en apparence ou en réalité) de manière à le comprendre ${ }^{37}$ ", rappelait Piaget, qui ajoutait : « La réfutation du réductionnisme qui doit permettre de "copier le réel" entraîne un appel au constructivisme ${ }^{38}$ ".

Appel qui a suscité la restauration et le déploiement contemporain du paradigme des épistémologies constructivistes, que les sciences de l'éducation, notamment anglo-saxonnes, ont trop souvent réduites à des considérations méthodologiques, sans se soucier de leur légitimation. À la différence des chercheurs, souvent francophones, qui ont su écouter et interpréter cet appel dans leurs expériences tant dans les domaines des sciences de l'éducation que dans tous les autres, ce qui les a conduits à mettre en valeur l'émerveillante capacité de l'esprit humain à enrichir plutôt qu'à appauvrir les représentations de ses expériences.

Valéry sait nous dire cet émerveillement : "Un nom manque à cette créature de pensée pour contenir l'expansion de termes trop éloignés d'ordinaire et qui se déroberaient. Aucun ne me parait plus convenir que

36 Paul Valéry, dans un article publié en 1939, consacré à « l'œuvre écrite de Léonard de Vinci ", dans Vues, Paris, La table ronde, 1947, p. 228.

37 Jean Piaget, Biologie et connaissance. Essai sur les relations entre les régulations organiques et les processus cognitifs, Paris, Gallimard, coll. «Idées », [1967] 1973, p. 22. 
celui de Léonard de Vincis ${ }^{39}$ ". Cette faculté de conjoindre intentionnellement, par projet délibéré, le phénomène auquel on prête attention, à quelques contextes, ne nous est-elle pas familière? Pourquoi nous faudrait-il nous l'interdire systématiquement sous le prétexte d'une « austère censure posée par le postulat d'objectivité... (tenu pour) consubstantiel à la science, postulat pur, à jamais indémontrable $e^{40} » ?$

Est-il judicieux pour connaitre et comprendre l'arbre, de le réduire d'abord et apriori en un tas de sciure ( le décomposer en autant de parcelles qu'il se pourrait ") ? Ne pouvons-nous l'entendre dans et par ses interactions " avec ce fond sur lequel il se détache »? Aristote dans ses Topiques s'est attaché à nous le rappeler. Et Vico nous rappela la puissance de « cette étrange capacité de l'esprit humain qui est de conjoindre » (capacité qu'il nommait l'ingenium, s'étonnant que la langue française n'ait pas su former d'équivalent satisfaisant pour traduire ce mot latin que nous légua Cicéron). Car « la Méthode (celle du discours cartésien) nuit à l'ingenium... et l'ingenium a été donné aux humains pour comprendre, c'est-à-dire pour faire ».

Dès lors que nous nous attachons à comprendre et à connaître d'abord par conjonction (par l'exercice de l'ingenium) plutôt que par disjonction (par exercice de l'analyse), autrement dit en ne craignant pas de commencer par complexifier (ou par enrichir nos représentations), nous pouvons reconsidérer les fonctions de la recherche et de l'enseignement, en assumant lucidement, en citoyens responsables, les risques épistémiques et éthiques du métier.

\section{Approcher aujourd'hui dans des termes nouveaux la question de la complexité}

L'attitude, l'esprit scientifique, ce n'est pas croire, mais penser ${ }^{41}$.

Les « lecteurs pensifs » souhaiteront poursuivre une méditation qui ne s'arrête pas à cet examen stimulant de la faisabilité de la critique

30) Paul Valéry, op. cit., p. 156.

40. Jacques Monod, Le basard et la nécessité. Essai sur la philosopbie naturelle de la biologie modeme, Paris, Seuil, 1970, p. 33.

41 Jean-Marie Lehn, dans Courrier du CNRS, 1990. 
épistémologique interne des connaissances scientifiques qu'ils construisent et enseignent.

Car nous pouvons continuer à pratiquer ces exercices en nous interrogeant sur notre aptitude à concevoir, sous la forme de modèles faits d'artefacts symboliques, nos « projets de perception ou d'attention ».

Ne pouvons-nous " apprendre à modéliser », comme savait le faire Léonard de Vinci pratiquant le Disegno ${ }^{42}$, et devons-nous toujours nous résigner à appliquer des modèles de nos perceptions du monde fait avant, et ailleurs, en d'autres contextes ? Sommes-nous condamnés à n'être que des abeilles dans leur ruche reproduisant le modèle géométriquement parfait de leur cellule de cire? Ne pouvons-nous être aussi architectes, capables de concevoir, ici et maintenant, les modèles de nos démarches projectives, construisant consciemment, et en délibérant ${ }^{43}$, nos chemins en marchant, sans que le modèle d'un but ultime unique à atteindre à tout prix nous soit définitivement imposé ? «Le mot construction que j'ai employé à dessein, pour désigner plus fortement le problème de l'intervention humaine dans les choses du monde, $[. .$.$] ce mot prend maintenant sa signification$ restreinte. L'architecture devient notre exemple ${ }^{44}$ ».

Il nous faut aussi le poursuivre en ne négligeant pas les responsabilités éthiques que toute critique épistémologique rappelle à la société. L'effet le plus pervers du «funeste présent » que nous lèguent les épistémologies positivistes et scientistes a été de réduire le «moralement bon au (présumé) scientifiquement vrai » dans nos cultures. Si bien que lorsqu'elles prennent conscience de cette fallace (OGM ou stockage des déchets radio-actifs

42 « Je disegno est d'une excellence telle qu'il ne fait pas que montrer les oeuvres de la nature, mais qu'il en produit des formes infiniment plus variées [...] Les œuvres que l'œil exige des mains de l'homme sont illimitées " (Léonard de Vinci, Codex urbanum, f. 502, 1162). On trouvera dans Le constructivisme (tome 1, Les enracinements, Paris, L'Harmattan, coll. «Ingenium », p. 87-89) un développement sur cet argument et ses références. Voir aussi Un art paradoxal. La notion de disegno en Italie (XIr-XVT siècles) (Paris, L'Harmattan, 2004, présenté à

http:/ /www.mcxapc.org/cahier.php?a=display\&ID=642).

4.3 Mais savons-nous délibérer ? Depuis la suppression de l'enseignement de la rhétorique dans la plupart de nos enseignements nous ne savons pas, et nous ne pouvons que nous affronter en des choix binaires. La récente et bienvenue restauration du concept de « démocratie délibérative » incitera-t-elle nos cultures à restaurer "cette antique sagesse » qui privilégie "l'éthique de la délibération »?

44 Paul Valéry, op. cit., p. 1188. 
aidant, si l'on ose dire !) les sociétés sont tentées de jeter le bébé de la critique épistémologique interne avec l'eau du bain scientiste-positiviste, ce qui nous priverait de notre capacité de diagnostic et d'élucidation collective des enjeux dont sont porteurs les «nouvelles connaissances » dès lors que nos cultures ne peuvent plus se les approprier. Il est fort compréhensible que les chercheurs scientifiques jouissent, grâce à la société qui les finance, du «bonheur de la recherche " ('aventure de la connaissance, aventure infinie). Mais doivent-ils prétendre que, ce faisant, ils ouvrent certainement à l'humanité les voies de «la recherche du bonheur»? Est-il admissible que l'on puisse aujourd'hui être chercheur scientifique ou enseignant sans avoir à s'interroger en permanence sur la légitimité socioculturelle et épistémique des connaissances que l'on produit et que l'on transmet? Aussi longtemps que les scientifiques ne s'attacheront pas à aviver leur propre culture épistémique et civique, ils ne pourront pas reprocher à leurs concitoyens leur manque de culture scientifique, qu'elle concerne les sciences dures ou les sciences douces.

Une fois encore cet exercice est fort « normalement » praticable. Il ne requiert que cette probité intellectuelle et cette conscience de la responsabilité civique que chacun attend de tout enseignant-chercheur dans une société démocratique. Le lecteur pensif en conviendra peut-être en lisant ces quelques lignes de conclusion que j'extrais de la Charte 2002 de la plus importante des institutions de recherche scientifique française (Le projet d'établissement 2002 du CNRS français) :

La société et la communauté des chercheurs partagent aujourd'hui un sentiment fort de vivre un moment de « révolution scientifique ». [...] Cet ébranlement, qui transforme à la fois les manières de concevoir et de faire la recherche et les conditions de son institutionnalisation, trouve son origine dans la nécessité qui s'impose aujourd'hui d'approcher dans des termes nouveaux la question de la complexité. [...]

S'attacher à la complexité, c'est introduire une certaine manière de traiter le réel et définir un rapport particulier à l'objet, rapport qui vaut dans chaque domaine de la science, de la cosmologie à la biologie des molécules, de l'informatique à la sociologie.

C'est reconnaitre que la modélisation se construit comme un point de vue pris sur le réel, à partir duquel un travail de mise en ordre, partiel et continuellement remaniable, peut être mis en œuvre.

Dans cette perspective, l'exploration de la complexité se présente comme le projet de maintenir ouverte en permanence, dans le travail d'explication scientifique lui-même, la reconnaissance de la dimension de l'imprédictibilité. 


\section{Bibliographie}

Bachelard, Gaston, Le nouvel esprit scientifique, Paris, PUF, 1934.

Barel, Yves, Le paradoxe et le système. Dix ans après, Grenoble, Presses universitaires de Grenoble, 1989.

Canto-Sperber, Monique (dir.), Les paradoxes de la connaissance. Essai sur le Menon de Platon, Paris, Odile Jacob, 1991.

Centre national de la recherche scientifique (CNRS), Construire une politique scientifique, 15 pages, à http://www.cnrs.fr/strategie/projet.html.

Comte, Auguste, "Appel aux conservateurs ", dans Du ponvoir spirituel (choix de textes de Pierre Arnaud), Paris, Le livre de poche, [1855] 1978, p. 493-494.

Descartes, René, Discours de la méthode, Paris, Libraire philosophique J. Vrin, [1637] 1970.

Dupuy, Jean-Pierre, Ordre et désordres. Enquête sur un nouveau paradigme, Paris, Seuil, 1982.

Jouven, Claude, «XXI" siècle : le retour du bon sens, mobiliser les hommes, c'est simplifier les systèmes », Le Monde, 8 avril 1986.

Kourilsky, François (coord.), Ingénierie de linterdisciplinarité. Un nouvel esprit scientifique, Paris, L'Harmattan, Collection Ingenium, 2002.

I. Blond, Jean Marie, Logique et méthode chez Aristote : étude sur la recherche des principes dans la pbysique aristotélicienne, Paris, Vrin, 1970 [1939].

Lehn, Jean-Marie, Coumier du CNRS, 1990.

Le Moigne, Jean-Louis, Le constructivisme, tome 1, Les enracinements, Paris, L'Harmattan, coll. «Ingenium », 1994.

Le Moigne, Jean-Louis, Le constructivisme, tome 3, Modéliser pour comprendre, Paris, L'Harmattan, 2004.

Le Moigne, Jean-Louis, «Sur un exceptionnel manifeste épistémologique : Symbols and Search. Merveilleuse et pourtant compréhensible est la computation (le traitement) heuristique des systèmes de symboles physiques $", L$ a revue d'intelligence artificielle, numéro spécial en hommage à H. A. Simon, Représentations, déconvertes et rationalité), Jacques Pitrat (dir.), Cachan, Hermès-Lavoisier, RSTI, série RIA, vol. 16, n ${ }^{(x)} 1-2,2002$, p. 251-268.

Le Moigne, Jean-Louis, Un ant paradoxal. La notion de disegno en Italie (XV'-XVT'siècle), Paris, L'Harmattan, 2004, http://www.mcxapc.org/cahier.php?a=display\&ID $=642$.

Luglio, Davide, La science nouvelle on l'extase de l'ordre. Connaissance, rhétorique et science dans l'cuure de G. B. Vico, Paris, PUF, 2003.

Marx, Karl, Le capital, dans CEures, tome 1, Économie, Paris, Gallimard, coll. « La pléiade », [1867] 1963.

Monod, Jacques, Le hasard et la nécessité. Essai sur la pbilosophie naturelle de la biologie moderne, Paris, Seuil, 1970.

Morin, Edgar, La métbode, tome 3, La connaissance de la connaissance, Paris, Seuil, 1986.

Morin, Edgar et Jean-Louis Le Moigne, L'intelligence de la complexité, Paris, L'Harmattan, 1999.

Pascal, Blaise, Pensées, 200-347 H3, Paris, Seuil, 1963.

Piaget, Jean, "Logique et connaissance scientifique », Encyclopédie Plëiade, 1967. 


\section{JEAN-LOUIS LE MOIGNE / SUR LA MÉTHODETOPICO-CRTTQUE...}

Piaget, Jean, Biologie et connaissance. Essai sur les relations entre les régulations organiques et les processus cognitifs, Paris, Gallimard, coll. « Idées », [1967] 1973.

Piaget, Jean, L'épistémologie génétique, Paris, PUF, coll. «Que Sais-je ? », 1972.

Platon, "Ménon ou de la vertu ", in CEumres complètes, Paris, Gallimard, coll. " I a pléiade ", 1990.

Prigogine, Ilya, La fin des certitudes, Paris, Odile Jacob, 1996.

Thom, René, La querelle du déterminisme, Paris, Gallimard, coll. « Le débat », 1990.

Valéry, Paul, Cabiers 1894-1914, tome 2, Paris, Gallimard, coll. « La pléiade », 1974.

Valéry, Paul, «Introduction à la méthode de Iéonard de Vinci ", dans CEuvres complètes, tome 1, Paris, Gallimard, coll. «Ia pléiade », [1894] 1974.

Valéry, Paul, V'ues, Paris, La table ronde, [1939] 1947.

Vico, Giambattista, De l'antique sagesse de l'Italie, traduction de Jules Michelet, Paris, Garnier Flammarion, [1710] 1993. 